\title{
BMJ Open Domains of health-related quality of life in age-related macular degeneration: a qualitative study in the Chinese cultural context
}

\author{
Wei Bian, ${ }^{1,2}$ Junli Wan, ${ }^{1,2}$ Graeme Smith, ${ }^{3}$ Shiying Li, ${ }^{1,2}$ Mingqiong Tan, ${ }^{1,2}$ \\ Fengjiao Zhou $^{1,2}$
}

To cite: Bian W, Wan J, Smith G, et al. Domains of health-related quality of life in age-related macular degeneration: a qualitative study in the Chinese cultural context. BMJ Open 2018;8:e018756. doi:10.1136/ bmjopen-2017-018756

- Prepublication history for this paper is available online. To view these files, please visit the journal online (http://dx.doi. org/10.1136/bmjopen-2017018756).

Received 20 July 2017 Revised 19 January 2018 Accepted 15 February 2018
Check for updates

${ }^{1}$ Southwest Eye Hospital, Third Military Medical University (Army Medical University), Chongqing, China

${ }^{2}$ Key Lab of Visual Damage and Regeneration \& Restoration of Chongqing, Chongqing, China ${ }^{3}$ Faculty School of Health and Social Care of Health and Life Science, Edinburgh Napier University, Edinburgh, UK

Correspondence to Wei Bian; 664091353@qq.com

\section{ABSTRACT}

Objective To explore which areas of health-related quality of life were affected in Chinese patients, and to identify whether the areas are well covered by validated questionnaires.

Design A qualitative study based on semistructured interviews was conducted. A qualitative thematic analysis following the approach of Colaizzi was used to analyse the interview data for significant statements and phrases. The themes and subthemes organised from the analysis were then compared by using the following current instruments: National Eye Institute Visual Function Questionnaire (NEIVFQ-25), Macular Disease Quality of life Questionnaire (MacDQoL) and Low-Luminance Questionnaire (LLD). Participants and setting Twenty-one patients with agerelated macular degeneration were recruited from the eye clinic of Southwest Eye Hospital in Chongqing, mainland China.

Results The mean age of the participants was 69.8 years (range 57-82 years) and the duration of the disease ranged from 3 months to 6 years. The qualitative analysis revealed nine important domains including symptoms, difficulties with daily activities, depending on others, depression and uncertainty, optimism and hope, social isolation, role change, family support and financial burden. However, all the three questionnaires were insufficient to capture the full extent of quality of life issues of Chinese patients with AMD, and MacDQoL covered more domains when compared with NEI-VFQ-25 and LLD.

Conclusion The domains of concepts important to people with AMD in the Chinese culture are not fully represented in the three widely used questionnaires. Nine important domains were identified for the assessment of quality of life and should be considered when assessing the impact of AMD on Chinese individuals. Further studies are needed to develop an AMD quality of life questionnaire, better tailored to the needs and culture of Chinese patients.

\section{INTRODUCTION}

Age-related macular degeneration (AMD) is currently the leading cause of irreversible visual impairment and blindness in developed countries, particularly among people older than 60 years. Approximately 30 million
Strengths and limitations of this study

- This is the first study to explore the influence of life experience and perception of Chinese patients on how age-related macular degeneration (AMD) has affected their health-related quality of life (HRQoL) by using qualitative methodology, and to identify whether the areas of concern and applicability addressed by patients are well covered by the existing HRQoL questionnaires.

-We compared three instruments with the findings of our qualitative study. Other vision-specific tools are not included in the present study.

- The samples in our study are not representatives for all the patients with AMD in China or elsewhere.

people are currently affected, accounting for $8.7 \%$ of the population worldwide, with estimates suggesting this number may increase to 288 million by $2040 .{ }^{1}$ AMD may lead to visual impairment including loss of central vision, decreased contrast sensitivity and metamorphopsia, causing difficulties with activities of daily living, such as reading, driving, crossing the street and recognising people..$^{2-5}$ Furthermore, AMD may be associated with reduced life satisfaction, anxiety, depression and social isolation affecting the health-related quality of life (HRQoL) of patients. ${ }^{6-8}$ Although, vascular endothelial growth factor inhibitor (anti-VEGF) may be used to halt or reduce disease progression and further risk of wet AMD, visual impairment usually persists after treatment. ${ }^{9}$

Presently, evaluation of HRQoL is increasingly popular in research and clinical settings. WHO has suggested that six fundamental dimensions should be applied to HRQoL testing including physical impact, psychological functioning, social and role activities, overall life satisfaction, and perceptions of health status. ${ }^{10}$ To better understand 
and quantify HRQoL of patients with AMD, a number of vision-specific and disease-specific questionnaires have been developed and applied, such as the National Eye Institute Visual Function Questionnaire (NEI-VFQ25), ${ }^{11}$ Macular Disease Quality of life Questionnaire $(\mathrm{MacDQoL})^{12}$ and Low-Luminance Questionnaire (LLD). ${ }^{13}$ NEI-VFQ-25 is most widely used to evaluate influencing factors and the effectiveness of treatment outcomes among patients with AMD.${ }^{3514}$ However, since it is a vision-specific instrument that can be applied to all kinds of eye diseases, it may not be as sensitive to symptoms such as central vision loss or emotional and spiritual disorders as a disease-specific instrument is. ${ }^{15} \mathrm{MacDQ}$ LL and LLD are designed specifically for patients with AMD. However, MacDQoL has been found to have serious psychometric shortcomings by the Rasch analysis, ${ }^{16}$ and LLD is only used to assess vision problems under low luminance in age-related maculopathy; therefore their application is limited. Furthermore, none of the three instruments addresses ethical and cultural differences in the analysis of different populations and none has been retrospectively evaluated to determine the accuracy of HRQoL assessments, or the sensitivity in detecting problems specific to Chinese patients with AMD.

In order to resolve this issue, it is necessary to fully understand the problems that patients with AMD have experienced from their own perspectives. The qualitative research is considered most appropriate to explore the experience and views of patients in-depth and to give the meaning through the systematic procedure.

Until now, there have been some qualitative research studies on patients with AMD, but these patients have been conducted but have focused on a single population such as older men or women, ${ }^{17}{ }^{18}$ or on individual issues such as impact on activities of daily living, ${ }^{19}$ emotional issues ${ }^{20}$ and psychosocial adjustment, ${ }^{21}$ or on the effects of new treatments such as anti-VEGF injection and reading rehabilitation. ${ }^{42}$ Most of the existing instruments are not comprehensive in assessing the HRQoL of patients with AMD patients . For example, transportation and independent driving are not mentioned and should be added to in the MacDQoL. ${ }^{23}$ Additionally, there are no studies that have comprehensively focused on the HRQoL influenced by AMD from the patients' own perspectives in the Chinese cultural context. For instance, ba-ba dancing is a very popular activity in China and plays an important part in daily life especially of elderly women. The HRQoL will be affected if the patients give up ba-ba dancing because of poor vision. Besides, the perception of family relationships may also vary in different cultures, resulting in different influences on emotional and psychological wellbeing. ${ }^{24}$ Chinese patients with AMD patients are usually cared for by spouses and relatives, and with lesser involvement of from healthcare professionals and rehabilitation institutions than in some other developed countries. The differences between the burden caused by care from family members and that from professional caregivers will exert different impacts on the HRQoL of the patients. ${ }^{25}$
The purpose of this study is to explore the influence of life experience and perception of Chinese patients on how AMD has affected their HRQoL by using qualitative methodology, and to identify whether the areas of concerns are adequately addressed by the existing QoL instruments including NEI-VFQ-25, MacDQoL and LLD. All the domains presented in this qualitative study can help develop a Chinese-specific instrument that can provide accurate information to understand the condition itself and to evaluate the effectiveness of the treatment.

\section{METHODS}

\section{Design and setting}

This was a descriptive qualitative study to explore the effects of AMD on the physical, psychological, emotional and social well-being of Chinese patients. The research was conducted in the eye clinic of Southwest Hospital (Chongqing, China), and was presented consistently with the checklist of Consolidated Criteria for Reporting Qualitative Research.

\section{Participant recruitment}

Purposive and convenience sampling was used to recruit patients from the eye clinic from May 2015 to May 2017. Patients meeting the following criteria were enrolled into the study: surgically diagnosed with AMD by the ophthalmologist; more than 45 years old; Chinese speaking and willing to participate in the study; without any other diseases that could affect the vision such as high myopia, macular splitting crack, diabetic retinopathy and retinal detachment; without any other ophthalmic treatment therapies or surgeries such as cataract, and glaucoma surgery; without any cognitive or intellectual impairment that could affect oral communication.

\section{Data collection}

Semistructured interviews were conducted face to face by the first and the second authors of this manuscript with qualitative research training and experience. Before the interviews, patients had completed an answer sheet containing demographic and clinical characteristics, purpose of the study and informed consent. Participants were informed that they could reject or withdraw from the research at any time or stage if they felt uncomfortable; each participant was anonymous; all the materials were privately kept in a locked cabinet and were only used for this research.

The interviews, ranging between $30 \mathrm{~min}$ and $60 \mathrm{~min}$, were carried out in a private and quiet room of the eye clinic. The interviewers attempted to remain neutral on what the participants said, and tried to be a sensitive and unbiased audience. Interviews started with general questions such as 'Tell me about your experiences and feelings about AMD'. Patients with AMD were asked questions on experiences of HRQoL without interference by anyone or any other kind of interference. Probing questions, such as 'Could you tell me more about it?' and 'What does that 
mean to you?' were asked to clarify the meaning of the responses and the detailed information during the interview. Also, emotional changes and body language were observed during the interview, supplemented by simple scene notes. Recruitment was stopped when the data were saturated.

\section{Data analysis}

The interview transcripts were audio recorded and transcribed verbatim by the second author. Transcripts were imported into the qualitative software analysis package NVivo V.10.0, and were coded by the first and the second authors, independently. All the transcripts were checked to ensure accuracy. Data analysis started with the first interview and was performed simultaneously with data collection.

A qualitative thematic analysis was conducted to analyse the interview data, following the approach of Colaizzi. ${ }^{26}$ Colaizzi's approach focuses on the feelings of the subjects and is highly inductive in analysing the data for significant statements and phrases. This analysis has seven stages: (1) reading the transcripts repeatedly and carefully while listening to the digital recordings; (2) extracting significant statements and essential elements from the transcripts; (3) coding the extracted elements and statements; (4) arranging the formulated meanings into several theme clusters and categories; (5) ensuring that the detailed descriptions completely specified every extracted theme; (6) making sure that the statements reflected the essential structure of the phenomenon; (7) returning the transcripts to the subjects to obtain their views and to verify the authenticity of the results. The first and second authors analysed the data, compared the results and had discussions with the study team to ensure the credibility of the data when there were any differences. Final themes were determined after checking with the members. Saturation occurred when no further information in relation to the determined themes appeared during the research process.

Informed consent was obtained by all participants prior to conducting the study.

Letters of alphabets instead of true names were used to protect the confidentiality of the participants. Audio tapes and other documents were kept in a locked cabinet after each interview was transcribed and verified by checking the accuracy according to the tapes. All the data were only available to the researchers and would be destroyed by the interviewers at the end of the study. All the procedures were conducted in accordance with the Declaration of Helsinki.

\section{Mapping of themes and subthemes to the disease-specific instruments}

After the qualitative analysis was complete, the themes and subthemes that emerged were compared with items from two AMD-specific instruments and one vision-specific instrument to identify whether these instruments could fully reflect the HRQoL of patients with AMD. The
Table 1 Characteristics of patients with age-related macular degeneration (AMD) participating in the interviews $(n=21)$

\begin{tabular}{ll}
\hline Variables & \\
\hline $\begin{array}{l}\text { Mean age, years (range) } \\
\text { Duration of the disease, } \\
\text { months (range) }\end{array}$ & $69.8(57-82)$ \\
$\begin{array}{l}\text { Gender } \\
\text { Male }\end{array}$ & $7(33.2)$ \\
\hline Female & $14(66.67 \%)$ \\
\hline Education & \\
\hline Primary or lower & $9(42.86 \%)$ \\
\hline Secondary & $10(47.62 \%)$ \\
\hline Tertiary or higher & $2(9.52 \%)$ \\
\hline Marital status & \\
\hline Married & $21(100 \%)$ \\
\hline Not currently married & $0(0 \%)$ \\
\hline Eye affected by AMD & $13(61.90 \%)$ \\
\hline Unilateral & $8(38.10 \%)$ \\
\hline Bilateral & $1(4.76 \%)$ \\
\hline Status of best-corrected visual acuity \\
\hline Severe visual impairment & $5(23.81 \%)$ \\
\hline Moderately affected & $8(38.10 \%)$ \\
\hline Mild visual impairment & $7(33.33 \%)$ \\
\hline Good vision & \\
\hline
\end{tabular}

instruments included for comparison were MacDQoL, LLD and NEI-VFQ-25. Content gaps and differences were identified and recorded.

\section{RESULTS \\ Participant characteristics}

Twenty-one patients were recruited, with a mean age of 69.8 years $(\mathrm{SD}=7.65)$; the youngest was 57 years old, and the oldest was 82 years. Seven $(33.33 \%)$ were male. Two $(9.52 \%)$ had tertiary or higher education, and nine $(42.86 \%)$ had basic education. All of the patients were married. By the time of the interviews, all the participants had been diagnosed with AMD for 3 months to 6 years. Eight patients (38.10\%) had bilateral AMD, one (4.76\%) had severe visual impairment (visual acuity worse than $6 / 60$, or $20 / 200$ in the better eye), five $(23.81 \%$ ) were moderately affected (visual acuity of $6 / 24-6 / 60$, or 20/80-20/200 in the better eye), eight (38.10\%) endured mild visual impairment (visual acuity of $6 / 12-6 / 18$, or 20/40-20/60) and seven (33.33\%) had relatively good vision (visual acuity better than or equal to $6 / 9$ or $20 / 30$ in the better eye) ${ }^{27}$ Full details of participant characteristics are described in table 1. 


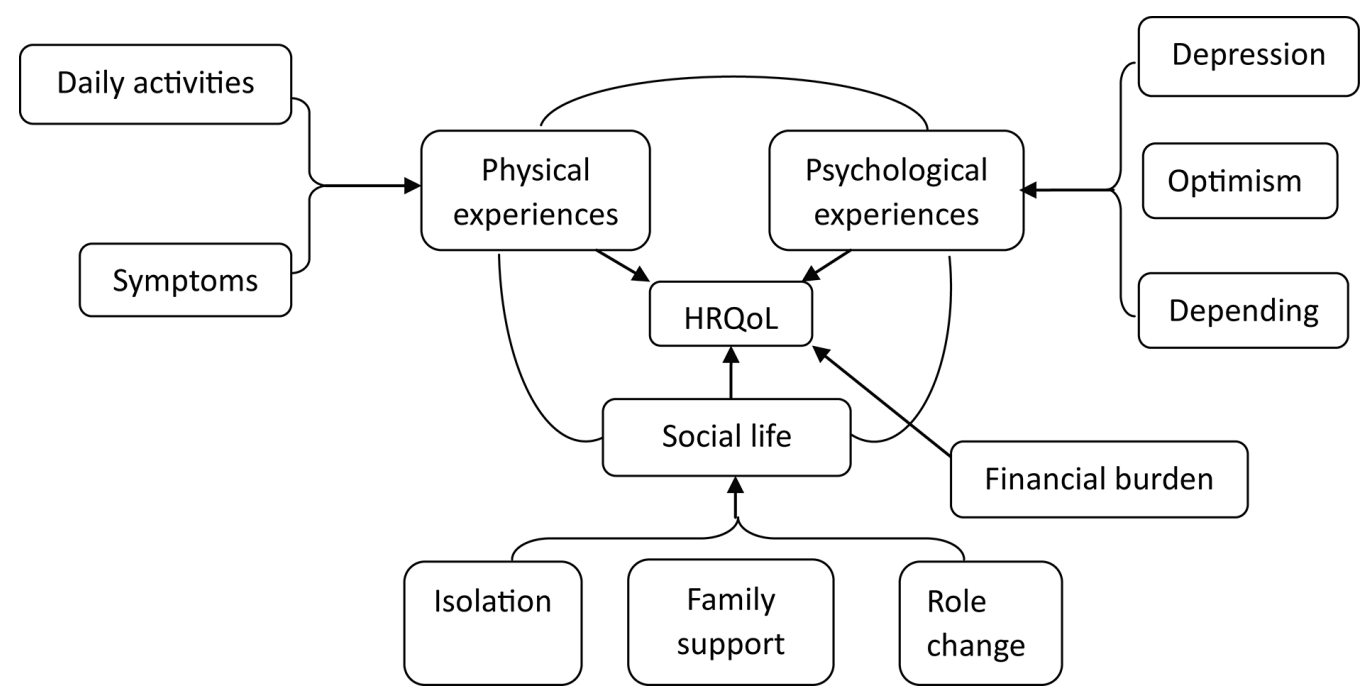

Figure 1 Domains of $\mathrm{HRQ}$ LL among patients with age-related macular degeneration. HRQoL, health-related quality of life.

\section{THEMES}

Three main themes concerning HRQoL of patients with AMD were identified based on the data: impact on physical experience, impact on psychological experience, and impact on social life and financial burden. The domains of the three themes are presented in detail in figure 1. Impact on physical experience described the effect of AMD on daily life including seeing, daily activities and depending on others. The psychological experiences included feelings of depression and hope, and also dependence on others. Although the physical and the psychological experiences were different, they always influenced and interacted with each other. Impact on social life included isolation from friends, neighbours and the wider community. In addition, the change of family role was a determining factor for sustaining normal life. Financial burden to the patients and their family was also a factor, and could influence the psychological health and social life of the patients.

\section{Impact on physical experience}

Symptoms

The the most common symptoms reported by the patients included blurred vision, central scotoma, metamorphopsia, diplopia, itch and dazzle light. In most cases, these symptoms could severely influence their self-management.

I can't distinguish whether it is sunny or rainy. I see everything's cloudy. I even couldn't see the passers and trees clearly.

I see anything with a black hole in the centre, out of shape and a little dark. When I look at the indicator light on the road, I see a circle of curved arrows.

\section{Difficulties with daily activities}

With progressive deterioration of visual function, most patients encountered many problems in their daily life. Routine activities like 'crossing a street', 'walking upstairs and downstairs', 'reading newspapers' or 'shopping in a supermarket' were considered challenging and needed extra effort and more time to perform.

Now I stay at home all day. I need someone to assist me in walking up and down stairs. Crutches are needed, and more attention should be paid to, when the road is rugged. I walk very slowly to avoid falling down.

I used to read books and newspapers every day. With the poor vision, it's very inconvenient to watch TV and read newspapers.

\section{Impact on psychological experience}

Depression and uncertainty

Most patients experienced psychological stress during the early stage of the illness. Some patients kept asking themselves. 'Why does it happen to me?' and 'Was I wrongly diagnosed?'. It was difficult for some to accept the disability.

In spite of the current success in treatment, patients were worried about the long-term effect of the treatment, 'Does it only work for a while?'. They were afraid that the condition would 'become worse', and that the 'disease may develop from unilateral to bilateral' and will eventually progress towards 'blindness'. Fear and worry about treatment and treatment efficacy was experienced and caused cautiousness and depression, also in relation to uncertainty about the future.

\section{Depending on others}

Unremitting difficulties with daily activities led to dependence on others. They had to rely on their friends and family members for helping them take public transport, fetch things or to take a bath.

I have to depend on my relatives when going to any places. I always ask my son to take me to the hospital although it will interfere with his work.

Although some participants found it easy to ask for help, the others found it inconvenient and embarrassing 
to depend on others. They did not want to be a trouble or burdening their families.

Most of the time, I really don't want to call for help. I feel like that I am rubbish and have to rely on others for everything. I have put too much burden on the family.

\section{Optimism and hope}

Some patients with exudative AMD treated with intravitreal anti-VEGF injection had an improvement in their visual function. They showed optimism and hoped for a positive outcome. Patients with vision that did not improve remained optimistic that the treatment would be effective.

It is not a terminal disease. I believe there is still a chance to improve my vision with the professional skills of doctors.

Thanks for god, I hope my vision improve greatly after the intravitreal anti-VEGF injection.

\section{Impact on social life}

Social isolation

Due to loss of independence and self-esteem, some patients stayed at home and were isolated from society. They were not able to participate in social activities such as dancing, travelling and having meals out with friends. Misunderstanding was reported due to patients not recognising acquaintances.

I was fond of baba-dancing and travelling with friends before, and I was a very active person. But now I don't go out for dancing or travelling, and spend less time with my friends and neighbours.

When acquaintances passed by, I couldn't see and say hello to them. I explained to them about my poor eye sight. However, they still thought that I was arrogant and pretended to do so. Now I seldom go out to avoid meeting them.

\section{Family support}

Many patients felt that family relationships became stronger as their visual function deteriorated. The family provided functional, emotional and economic support to the patients, such as "communicating with them', 'loving each other' and 'providing money for the treatment'. They helped the patients reduce the incidence of depression and other health problems, overcome various obstacles and integrated them into social life more effectively.

My husband does everything for me at home, and follows me everywhere. My family members often communicate with me with comfort and love, and encourage me to participate in activities for elderly people.

\section{Role change}

Many participants reported their family and social role had changed because of the visual disability, and they could no longer do things for their friends and families as before.

I was the breadwinner and in charge of everything in my family before, but now I am not relied on by others anymore.

\section{Financial burden}

The progression of wet AMD could be halted and vision could remain stable after treatment with anti-VEGF. However, the cost of the anti-VEGF injections was paid for by the patients and was a financial burden to the whole family.

It is a big financial pressure for a common family that anti-VEGF is paid all by myself. I have to borrow money from the relatives and friends. I have to wait for blindness if I can't afford it.

\section{Representation of AMD HRQOL domains}

Of the nine underlying domains identified in this qualitative study, eight were contained in at least one instrument of NEI-VFQ-25, MacDQoL and LLD (table 2). However, none of these questionnaires included items about optimism and hope. MacDQoL contained items related to family support and financial burden, but factors like symptoms, and optimism and hope for the treatment and future were not found. Furthermore, in NEI-VFQ-25 and LLD, a large number of items assessing the difficulties in driving did not involve those problems that were specific to the Chinese patients with AMD.

\section{DISCUSSION}

This qualitative study documents how AMD affects various aspects of daily life in Chinese patients. The nine important domains of HRQoL included: symptoms, difficulties with daily activities, depression and uncertainty, optimism and hope, depending on others, role change, social isolation, family support and financial burden. Difficulties with daily activities were the main problems reported by most patients with AMD, consistent with the findings of previous studies. ${ }^{17} 2829$ Patients frequently described feelings of depression due to their physical limitations, worry about vision loss and uncertainty about treatment and their life. Additionally, dependence on others, being misunderstood, and loss of self-esteem and isolation from society were reported.

This study highlighted the heavy psychological burden associated with AMD, in keeping with previous reports. ${ }^{20} 29$ Patients had negative emotions such as denying disease, depression and frustration due to disability in daily activities, being misunderstood by others, fearing to be a burden on the family, and experiencing many social barriers, especially in the early stage of the disease. In 
Table 2 Key domains of HRQoL in NEI-VFQ-25, MacDQoL and LLD

\begin{tabular}{|c|c|c|c|}
\hline Key domains of HRQoL & NEI-VFQ-25 items & MacDQoL items & LLD items \\
\hline \multicolumn{4}{|l|}{ Physical experiences } \\
\hline $\begin{array}{l}\text { Difficulties with daily } \\
\text { activities }\end{array}$ & $\begin{array}{l}\text { Difficulties with reading, seeing } \\
\text { objects, finding objects, going } \\
\text { downstairs, matching clothes, } \\
\text { going out to movies/plays, } \\
\text { accomplishing less, limited in } \\
\text { things that can be done }\end{array}$ & $\begin{array}{l}\text { Difficulties with household } \\
\text { tasks, personal affairs, } \\
\text { shopping, doing } \\
\text { things physically, getting } \\
\text { out, leisure, mishaps, having } \\
\text { meals, the time for doing } \\
\text { things }\end{array}$ & $\begin{array}{l}\text { Difficulties with reading } \\
\text { the menu, newspaper, material } \\
\text { Fall at night }\end{array}$ \\
\hline Symptoms & $\begin{array}{l}\text { Ocular pain, itch, burning } \\
\text { sensation }\end{array}$ & None & $\begin{array}{l}\text { Blurred vision } \\
\text { Poor colour vision, and night } \\
\text { and peripheral vision }\end{array}$ \\
\hline \multicolumn{4}{|l|}{ Emotional experiences } \\
\hline Depression and uncertainty & $\begin{array}{l}\text { Feeling frustrated, } \\
\text { embarrassment, no control, } \\
\text { Worry }\end{array}$ & $\begin{array}{l}\text { Losing self-confidence and } \\
\text { motivation } \\
\text { Worrying about the future }\end{array}$ & Feeling upset, depressed \\
\hline Depending on others & $\begin{array}{l}\text { Rely on others' words } \\
\text { Need help from others }\end{array}$ & Do things dependently & Depend on others for help \\
\hline \multicolumn{4}{|l|}{ Social life } \\
\hline Social isolation & $\begin{array}{l}\text { Seeing how people react } \\
\text { Visiting others } \\
\text { Staying at home most of time }\end{array}$ & $\begin{array}{l}\text { Less work opportunities } \\
\text { Worse personal relationship } \\
\text { and social life } \\
\text { More holiday } \\
\text { Better reaction from others }\end{array}$ & $\begin{array}{l}\text { Difficulty seeing people's } \\
\text { faces, going out to night- } \\
\text { time social events, making } \\
\text { mistakes at social events }\end{array}$ \\
\hline Family support & None & Better family life & None \\
\hline Role change & Role limitation & Can't do things for others & $\begin{array}{l}\text { Can help others as much as } \\
\text { before }\end{array}$ \\
\hline
\end{tabular}

HRQoL, health-related quality of life; LLD, Low-Luminance Questionnaire; MacDQoL, Macular Disease Quality of life Questionnaire; NEIVFQ-25, National Eye Institute Visual Function Questionnaire.

those undergoing treatment there was anxiety about visual prognosis even if vision had been stable for many years. Three participants even mentioned thoughts of 'having meaningless life and living' or 'waiting for death in the nursing home'. They have been immersed in grief, and may become the victims of continuous depression, frustration and agony. However, psychological impact is a serious problem and has been neglected by most Chinese ophthalmological researchers. ${ }^{30}$ There were no interventions available despite reports of medical treatment and visual assistance to help Chinese patients with AMD. Thus, it is important to give urgent attention to this and to improve the rehabilitation system in China to help patients with AMD. Meanwhile, in contrast to past studies that reported control of AMD as 'false hope', our research found that some patients paid attention to disease progression, took a positive and optimistic attitude towards life, and adapted to the crisis. These abilities may assist them in recovering mental health and improve HRQoL. These comments are in line with the comments expressed by another qualitative study ${ }^{32}$ but all the three existing instruments do not include any of them.
The findings of our study revealed that family support was a key domain in QoL of patients with AMD, which highly reflected the traditional Chinese culture. In China, family played an important role in maintaining psychological health, and supporting the growth, survival and social development of patients. AMD was considered to be an important 'family affair'. The family oriented culture determined that the family centred care took over disease management instead of asking professional medical staff or a nursing home for help. Responsibility of patient care was almost always taken by family members, such as helping the patients with their daily life, taking them to the hospital and being involved in their rehabilitation. This involvement could provide emotional and social support, reduce the incidence of depression and mental health problems, help the patient cope with their visual impairment, and encourage normal family and social life. However, if the family did not function well, it might have an adverse impact on the lives of individuals. ${ }^{33}$ Therefore, medical staff should provide information and care for both patients and family members, because most medical decisions were made by the whole 
family, which was different from the situation in Western countries who advocate subjective intention of the patients. Furthermore, involving families in AMD rehabilitation, developing family centred projects and building social networking sites were important to provide effective information paths for the family, to help the family members integrate into interventions of the patients, and to achieve the goal of rehabilitation. ${ }^{34}$

The financial burden of AMD treatment was reported by many patients and was another subtheme. In the special medical system in China, all the cost of AMD treatment could not be reimbursed by medical insurance. Each anti-VEGF injection currently costs $\$ 1478$ and this is usually a big financial burden given that the average monthly salary in China is approximately $\$ 656$. The problem is exacerbated by the need for repeated antiVEGF injections. Many patients considered themselves a financial burden to the family, despaired of the future and gave up the treatment. This domain was a significant index that could assess the current economic difficulties of the patients and encourage them to get appropriate interventions according to their financial status.

It is interesting to note that difficulties in driving were not mentioned by the patients. In China, driving is not as common as in many other countries, especially in older people. Many patients with AMD did not possess a driver's license and never considered it a problem, because learning to drive was considered a luxury when they were younger. In Western countries, driving is common in daily life and having to give up driving has been reported as causing inconvenience, greater dependence on others and a feeling of being useless. ${ }^{29}$ As driving is a less common feature of daily life in China it may be less relevant to the AMD HRQoL questionnaire.

Overall, MacDQoL covered more domains except for symptoms, optimism and hope, compared with the other two questionnaires. However, the multiplicative rating scale of MacDQoL is found to have flaws and does not provide scientific measurement of HRQoL. ${ }^{16}$ NEI-VFQ-25 is the most widely used instrument in evaluating HRQoL of Chinese patients with AMD. ${ }^{35-37}$ However, some domains like family support, financial burden, optimism and hope, significant to Chinese patients with AMD, were not well covered by NEI-VFQ25. So there were significant questions to consider regarding this instrument before we conducted the research. Is it the most appropriate instrument that can fully reflect HRQoL of patients with AMD, and can it become one of the indicators for assessing the effectiveness of interventions? The limitations of QoL issues relating to AMD in paper-pencil questionnaires were recently highlighted by the published paper with regard to disease-specific ophthalmic instruments-the eye-tem bank project. ${ }^{38}$ This project aims to develop item banks and computerised adaptive testing instruments for all major eye diseases, including AMD. It uses Rasch analysis to develop and validate the instruments which can make them more precise and responsive than the traditional methods of psychometric assessment. Furthermore, the item bank implemented via computerised adaptive testing can provide comprehensive, rapid, dynamic, precise and accurate patient-reported outcome measures of HRQoL. We found that themes like 'financial burden' and 'family support' in our study could also be found in the HRQoL domains of the eye-tem bank. It is possible that the eye-tem bank method could form the basis of a modified AMD HRQoL questionnaire better adapted to the needs and concerns of Chinese patients.

There are a few limitations of our study. First, the sample was relatively small and specific to our eye clinic and did not incorporate a wider range of patients with AMD from different areas and institutions, so the results may vary with regard to different hospitals or communities. Second, all the interviews were conducted by the first and the second authors of this manuscript. Their philosophy, personal experience and cultural background might influence the collection and analysis of the data. However, the whole research was conducted under the guidance of the qualitative research experts, and group discussion was held to ensure the rigour when different opinions occurred. Third, we compared only three instruments with the findings of our qualitative study. Other vision-specific tools like the Impact of Vision Impairment, Visual Function Index and Low Vision Quality of life Questionnaire were not included in the present study. However, NEI-VFQ-25 is the most classic and widely used vision-specific tool in AMD and other eye diseases with low vision. MacDQoL and LLD are the AMD-specific tools that are considered to be more sensitive than the generic instrument. ${ }^{39}$ All of the three instruments are widely used, and could be the preventives of current paper-pencil questionnaires for assessing QoL of patients with AMD.

In conclusion, HRQoL of patients with AMD in China is influenced by Chinese culture. We identified physical experience, impact on psychological experience, impact on social life and financial burden in this qualitative study. The subthemes including family support and financial burden were highlighted in the Chinese context. There were no appropriate paper-pencil questionnaires for assessing QoL of the Chinese patients with AMD. Thus, it is very important for medical staff to develop an appropriate instrument for assessing quality of life (QOL) of patients with AMD accurately, and provide suitable physical, psychological, emotional and social care based on the results. Further studies are needed to develop an AMD questionnaire specific to Chinese patients to assess HRQoL of Chinese patients with AMD in clinical trials.

Acknowledgements The authors thank the participants who volunteered to take part in this research. The authors also thank Professor Anthony Robson and Miss Jia Wang for help in modifying the language of this manuscript.

Contributors WB, GS and JW contributed to the conception and design of the study; WB, JW and SL were responsible for acquisition of data; WB, JW, MT and FZ were responsible for the analysis of the study and interpretation of data; WB and GS drafted the article; WB, GS, JW, SL, MT and FZ revised the article critically for important intellectual content; GS copy-edited the manuscript. 
Funding This study was funded by the Army Medical University of Humanities and Social Science project (2017XRW21) and the First Affiliated Hospital of Third Military Medical University Innovation Research Grants (SWH2015QN04).

Competing interests None declared.

Patient consent Obtained.

Ethics approval Ethics Committee of the First Affiliated Hospital of Third Military Medical University, PLA (Ethics Reference S13/05/109).

Provenance and peer review Not commissioned; externally peer reviewed.

Data sharing statement There are no additional data available.

Open Access This is an Open Access article distributed in accordance with the Creative Commons Attribution Non Commercial (CC BY-NC 4.0) license, which permits others to distribute, remix, adapt, build upon this work non-commercially, and license their derivative works on different terms, provided the original work is properly cited and the use is non-commercial. See: http://creativecommons.org/ licenses/by-nc/4.0/

(c) Article author(s) (or their employer(s) unless otherwise stated in the text of the article) 2018. All rights reserved. No commercial use is permitted unless otherwise expressly granted.

\section{REFERENCES}

1. Wong WL, Su X, Li X, et al. Global prevalence of age-related macular degeneration and disease burden projection for 2020 and 2040: a systematic review and meta-analysis. Lancet Glob Health 2014;2:e106-e116.

2. Šiaudvytytė L, Mitkutė D, Balčiūnienè J. Quality of life in patients with age-related macular degeneration. Medicina 2012;48:109-11.

3. Matamoros E, Maurel F, Léon N, et al. Quality of Life in Patients Suffering from Active Exudative Age-Related Macular Degeneration: The EQUADE Study. Ophthalmologica 2015;234:151-9.

4. McCloud C, Khadka J, Gilhotra JS, Pesudovs K, et al. Divergence in the lived experience of people with macular Degeneration. Optometry and Vision Science 2014;91:966-74.

5. Qiu M, Wang SY, Singh K, et al. Association between visual field defects and quality of life in the United States. Ophthalmology 2014;121:733-40.

6. Jivraj J, Jivraj I, Tennant M, et al. Prevalence and impact of depressive symptoms in patients with age-related macular degeneration. Can J Ophthalmol 2013;48:269-73.

7. Mathew RS, Delbaere K, Lord SR, et al. Depressive symptoms and quality of life in people with age- related macular degeneration. Ophthalmic Physiol Opt 2011;31:375-80.

8. Senra H, Ali Z, Balaskas K, et al. Psychological impact of anti-VEGF treatments for wet macular degeneration-a review. Graefes Arch Clin Exp Ophthalmol 2016;254:1873-80.

9. Rovner BW, Casten RJ, Hegel MT, et al. Improving function in age-related macular degeneration: a randomized clinical trial. Ophthalmology 2013;120:1649-55.

10. Beaton DE, Schemitsch $E$. Measures of health-related quality of life and physical function. Clin Orthop Relat Res 2003;413:90-105.

11. Mangione CM, Lee PP, Gutierrez PR, et al. Development of the 25-item National Eye Institute Visual Function Questionnaire. Arch Ophthalmol 2001;119:1050-9.

12. Mitchell J, Wolffsohn JS, Woodcock A, et al. +Psychometric evaluation of the MacDQoL individualised measure of the impact of macular degeneration on quality of life. Health Qual Life Outcomes 2005;3:25.

13. Owsley C, McGwin G, Scilley K, et al. Development of a questionnaire to assess vision problems under low luminance in agerelated maculopathy. Invest Ophthalmol Vis Sci 2006;47:528-35.

14. Coleman AL, Yu F, Ensrud KE, et al. Impact of age-related macular degeneration on vision-specific quality of life: Follow-up from the 10year and 15-year visits of the Study of Osteoporotic Fractures. Am J Ophthalmol 2010;150:683-91.
15. Samsa G, Edelman D, Rothman ML, et al. Determining Clinically Important Differences in Health Status Measures. Pharmacoeconomics 1999;15:141-55.

16. Finger RP, Fenwick $\mathrm{E}$, Pesudovs $\mathrm{K}$, et al. Rasch analysis reveals problems with multiplicative scoring in the macular disease quality of life questionnaire. Ophthalmology 2012;119:2351-7.

17. Moore LW, Miller M. Older men's experiences of living with severe visual impairment. J Adv Nurs 2003;43:10-18.

18. Moore LW. Severe visual impairment in older women. West $J$ Nurs Res 2000;22:571-95.

19. Ivanoff SD, Sjöstrand J, Klepp KI, et al. Planning a health education programme for the elderly visually impaired person -- a focus group study. Disabil Rehabil 1996;18:515-22.

20. Scilley K, Dreer LE, Bray CR, et al. Focus Groups With Persons Who Have Age-Related Macular Degeneration: Emotional Issues. Rehabilitation Psychology 2006;51:23-9.

21. Stanford $\mathrm{P}$, Waterman $\mathrm{H}$, Russell WB, et al. Psychosocial adjustment in age related macular degeneration. Br J Vis Impair 2009;27:129-46.

22. Feely M, Vetere A, Myers LB. A qualitative analysis of reading rehabilitation of persons with age-related macular degeneration. $J$ Vis Impair Blind 2007;101:44-9.

23. Ord LM, Wright J, DeAngelis MM, et al. Quality of Life with Macular Degeneration Is Not as Dark as It May Seem: Patients' Perceptions of the MacDQoL Questionnaire. J Clin Med 2015;4:1841-52.

24. Mogk M. The difference that age makes: cultural factors that shape older adults' responses to age-related macular degeneration. $J$ Vis Impair Blind 2008;102:581-90.

25. Gohil R, Crosby-Nwaobi R, Forbes A, et al. Caregiver Burden in Patients Receiving Ranibizumab Therapy for Neovascular Age Related Macular Degeneration. PLoS One 2015;10:e0129361.

26. Colaizzi PF. Psychological research as the phenomenologist views it. In: Valle RS, King M, eds. Existential-phenomenological, 1978.

27. Wong EYH GR, Hassell JB, Keefe JE. The experience of age-related macular degeneration. J Vis Imp Blind 2004;98:629-40.

28. Ivanoff SD, Sjöstrand J, Klepp KI, et al. Planning a health education programme for the elderly visually impaired person--a focus group study. Disabil Rehabil 1996;18:515-22.

29. Wong EYH, Guymer RH, Hassell JB, et al. The experience of agerelated macular degeneration. J Vis Impair Blind 2004;98:629-40.

30. Sturrock BA, Xie J, Holloway EE, et al. The influence of coping on vision-related quality of life in patients with low vision: a prospective longitudinal study. Invest Ophthalmol Vis Sci 2015;56:2416.

31. Bennion AE, Shaw RL, Gibson JM. What do we know about the experience of age related macular degeneration? A systematic review and meta-synthesis of qualitative research. Soc Sci Med 2012;75:976-85.

32. McCloud C, Khadka J, Gilhotra JS, et al. Divergence in the lived experience of people with macular degeneration. Optom Vis Sci 2014;91:966-74.

33. Cimarolli, V, Boerner K. Social support and well-being in adults who are visually impaired. J Vis Impair Blind 2005;99:521-34.

34. Sörensen S, White K, Mak W, et al. The macular degeneration and aging study: design and research protocol of a randomized trial for a psychosocial intervention with macular degeneration patients. Contemp Clin Trials 2015;42:68-77.

35. Zhu W, Ren X, Yang X, et al. [A survey of vision-related quality of life in patients with exudative age-related macular degeneration]. Zhonghua Yan Ke Za Zhi 2014;50:189-93.

36. Huang YM, Dou HL, Huang FF, et al. Effect of supplemental lutein and zeaxanthin on serum, macular pigmentation, and visual performance in patients with early age-related macular degeneration. Biomed Res Int 2015;2015:1-8.

37. Wang LL, Liu WJ, Liu HY, et al. Single-site baseline and short-term outcomes of clinical characteristics and life quality evaluation of Chinese wet age-related macular degeneration patients in routine clinical practice. Chin Med J 2015;128:1154-9.

38. Khadka J, Fenwick E, Lamoureux E, et al. Methods to develop the eye-tem bank to measure ophthalmic quality of life. Optom Vis Sci 2016;93:1485-94.

39. Dixon P, Dakin H, Wordsworth S. Generic and disease-specific estimates of quality of life in macular degeneration: mapping the MacDQoL onto the EQ-5D-3L. Qual Life Res 2016;25:935-45. 